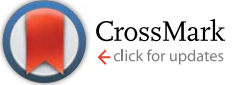

Cite this: RSC Adv., 2015, 5, 12686
Received 20th December 2014 Accepted 13th January 2015

DOI: 10.1039/c4ra16749j

www.rsc.org/advances

\section{Synthesis of conjugated D-A polymers bearing bi(dithienogermole) as a new donor component and their applications to polymer solar cells and transistors $\dagger$}

\author{
Joji Ohshita, ${ }^{* a}$ Masayuki Miyazaki, ${ }^{a}$ Makoto Nakashima, ${ }^{a}$ Daiki Tanaka, ${ }^{a}$ \\ Yousuke Ooyama, ${ }^{a}$ Takuya Sasaki, ${ }^{b}$ Yoshihito Kunugi ${ }^{b}$ and Yasushi Morihara ${ }^{c}$
}

\begin{abstract}
New donor-acceptor $\pi$-conjugated polymers composed of alternating bi(dithienogermole) (DTG2) and benzo-(BT) or pyridinothiadiazole (PT) units (pDTG2-BT or pDTG2-PT) were prepared and their optical, electrochemical, photovoltaic, and semiconducting properties were investigated. pDTG2-BT showed two absorption maxima at 738 and $686 \mathrm{~nm}$, respectively. Of the two, one at longer wavelength likely due to the aggregated polymer segments was relatively intensified, when the spectrum was measured in film. In contrast, pDTG2-PT showed one broad band at $686 \mathrm{~nm}$, which shifted to longer wavelength at 748 $\mathrm{nm}$ in film. These results indicate stronger interchain interaction for PDTG2-BT. Cyclic voltammograms of the polymer films showed pseudo-reversible profiles and the anodic peak of PDTG2-BT appeared at lower potential than that of PDTG2-PT, indicating the higher-lying HOMO (Highest Occupied Molecular Orbital) for pDTG2-BT. The polymer electronic states were also examined by the quantum chemical calculations on the polymer models. Of those polymers, pDTG2-BT exhibited the higher photovoltaic performance. The power conversion efficiency of the cell (ITO/PEDOT:PSS/pDTG2-BT:PC ${ }_{71} B M / L i F / A l$ ) reached $4.30 \%$ with $V_{\mathrm{oc}}=0.53 \mathrm{~V}, J_{\mathrm{sc}}=12.68 \mathrm{~mA} \mathrm{~cm}{ }^{-2}$, and $\mathrm{FF}=0.64$, presumably reflecting the stronger interchain interaction, although pDTG2-PT afforded higher $V_{\text {oc }}$, arising from its lower-lying HOMO. pDTG2-BT also exhibited higher performance as a thin film transistor material than pDTG2-PT. The performance of the cell with PDTG2-BT was also higher than that based on a similar alternating dithienogermole (DTG)-BT polymer, reported previously.
\end{abstract}

\section{Introduction}

Bulk heterojunction polymer solar cells (BHJ-PSCs) are of current interest because of their potential applications in lightweight and flexible modules and simple fabrication by a solution process. In this system, a blend film consisting of an electron-donating host polymer and an electron-accepting guest material, such as PCBM, is employed as the active layer. In this area, conjugated donor-acceptor (D-A) polymers have been extensively studied as the host polymers. ${ }^{1}$ The broad and red-

\footnotetext{
${ }^{a}$ Department of Applied Chemistry, Graduate School of Engineering, Hiroshima University, Higashi-Hiroshima 739-8527, Japan. E-mail: jo@hiroshima-u.ac.jp ${ }^{b}$ Department of Applied Chemistry, Faculty of Engineering, Tokai University, 4-1-1 Kitakaname, Hiratsuka 259-1292, Japan

'Synthesis Research Laboratory, Kurashiki Research Center, Kuraray Co., Ltd., 2045-1 Sakazu, Kurashiki 710-0801, Japan

$\dagger$ Electronic supplementary information (ESI) available: Experimental procedure for monomer preparation, ${ }^{1} \mathrm{H}$ NMR spectra of pDTG2-BT and pDTG2-PT, XRD patterns and AFM images of the polymer films optimized geometry, and LUCO and HOCO profiles of model polymer PDTG2-PT0, and response curves of TFTs with pDTG2-BT and pDTG2-PT]. See DOI: 10.1039/c4ra16749j
}

shifted absorptions of $\mathrm{D}-\mathrm{A}$ polymers make it possible to utilize the wide range of wavelengths in sunlight. High carrier transporting properties of the polymer films are also desired for the host polymers.

On the other hand, dithienosilole (DTS) has been developed as an interesting $\pi$-conjugated system. Its highly planar structure and well-known $\sigma^{*}-\pi^{*}$ interaction between silicon $\sigma^{*}$ - and bithiophene $\pi^{*}$-orbital leads to extended conjugation. ${ }^{2}$ DTS has been extensively studied as a promising donor component of D-A polymers for BHJ-PSCs, ${ }^{3}$ since Yang and co-workers prepared a DTS-benzothiadiazole (BT) alternating copolymer (pDTS-BT R = 2-ethylhexyl in Chart 1) and demonstrated its efficient photovoltaic properties. ${ }^{3 a}$ Recently, we introduced dithienogermole (DTG) as a new donor unit, hypothesizing that the introduction of the heavier germanium atom in place of silicon would provide stronger chain-chain interactions to enhance the carrier mobility in the polymer film (pDTG-BT in Chart 1). ${ }^{4 a b}$ At nearly the same time, Reynolds et al. and Leclerc et al. also reported the synthesis of DTG-containing photovoltaic polymers. ${ }^{4 c, \boldsymbol{d}}$ As expected, some DTG-containing polymers exhibited superior photovoltaic performance in BHJ-PSC 


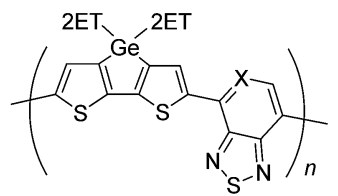

2ET = 2-ethylhexyl pDTG-BT $(X=\mathrm{CH})$ pDTG-PT $(X=N)$

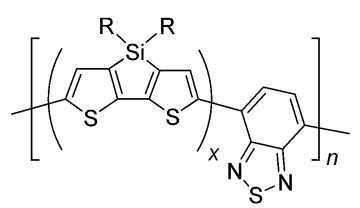

$\mathrm{R}=$ 2-ethylhexyl or $n$-octyl pDTS-BT $(x=1)$ pDTS2-BT $(x=2)$
Chart 1 DTG- and DTS-containing D-A polymers.

compared with the DTS analogues, primarily due to their higher current density. ${ }^{5}$ In the course of our studies to further improve the photovoltaic properties of DTG-containing polymers, we demonstrated that introduction of pyridino-thiadiazole (PT) in place of BT led to the enhanced photovoltaic properties (pDTGPT in Chart 1). ${ }^{6}$

Recently, a bi(dithienosilole)-benzothiadiazole alternating polymer (pDTS2-BT R $=n$-octyl) was prepared and it was found that pDTS2-BT exhibited improved thin film transistor (TFT) activity than that of pDTS-BT ( $\mathrm{R}=n$-octyl), likely due to the stronger $\pi-\pi$ stacking, enhancing the film hole-transporting properties (Chart 1). ${ }^{7}$ These results were suggestive of a potential of bidithienosilole as the efficient donor unit for the D-A host polymers that can be used as the host polymers in high performance BHJ-PSCs. However, similar approach has not been applied to DTG polymers. In this paper, we report the synthesis of new bi(dithienogermole) (DTG2)-BT and -PT alternating polymers and their optical, electrochemical, photovoltaic, and semiconducting properties.

\section{Experimental}

\section{General}

All reactions were carried out in dry argon. Diethyl ether and chlorobenzene that were used as the reaction solvents were distilled from calcium hydride and stored over activated molecular sieves before use. NMR spectra were recorded on a Varian System 500 spectrometer. UV-Vis spectra were measured with a Hitachi U-2910 spectrometer. Molecular weight of pDTG2-PT was determined relative to polystyrene standards by gel permeation chromatography (GPC) using THF as an eluent and serially connected Shodex KF2001 and KF2002 columns. Thermogravimetric analysis (TGA) was carried out on a SII TG/ DTA-6200 analyser under a gentle nitrogen flow of $30 \mathrm{~mL} \mathrm{~min}{ }^{-1}$ at a heating rate of $10{ }^{\circ} \mathrm{C} \mathrm{min}^{-1}$. Fabrication and evaluation of $\mathrm{BHJ}$-PSCs were performed as reported in the literature. ${ }^{6}$ Bottom contact TFTs were fabricated by spin-coating $0.4 \mathrm{wt} \%$ chlorobenzene solutions of the polymers on patterned substrates with integrated array electrodes $(d=210 \mathrm{~nm}, L=10 \mu \mathrm{m}, W=2 \mathrm{~cm})$, in a fashion similar to that reported in the literature. ${ }^{8}$

\section{Preparation of pDTG2-BT and pDTG2-PT}

A mixture of DTG2Sn (0.244 g, $0.195 \mathrm{mmol})$, dibromobenzothiadiazole $(0.057 \mathrm{~g}, 0.194 \mathrm{mmol}), \mathrm{Pd}_{2}(\mathrm{dba})_{3}(3.6 \mathrm{mg}, 0.0040$ $\mathrm{mmol}), \mathrm{P}(o-\mathrm{Tol})_{3}(6.0 \mathrm{mg}, 0.020 \mathrm{mmol}), \mathrm{CuO}(15.7 \mathrm{mg}, 0.197$ $\mathrm{mmol}$ ), and $15 \mathrm{~mL}$ of chorobenzene was heated to reflux for 5 days. The resulting precipitates were filtered and extracted with chlorobenzene. To remove the residual palladium and copper, the filtrate and the extract were combined and stirred at $80{ }^{\circ} \mathrm{C}$ for $2 \mathrm{~h}$ with $30 \mathrm{~mL}$ of an aqueous solution of sodium $N, N$ diethyldithiocarbamate trihydrate $(10 \mathrm{wt} \%)$. The organic layer was separated and washed with water, $3 \%$ acetic acid (aq), then water again. After drying the organic layer with anhydrous magnesium sulfate, the solvent was evaporated and the residue was precipitated from ethanol to provide a black solid. The solid was placed in a Soxhlet extractor and washed with hot methanol, ethyl acetate, and hexane, in this order. The resulting solid that was insoluble in those hot solvents was extracted with hot toluene. Polymer pDTG2-BT was obtained as a black solid by pouring the toluene extract to hexane (53 mg, 16\% yield): m.p. > $300{ }^{\circ} \mathrm{C},{ }^{1} \mathrm{H}$ NMR $\left(\delta\right.$ in $\left.\mathrm{C}_{6} \mathrm{D}_{4} \mathrm{Cl}_{2}, 500 \mathrm{MHz}\right), 8.59-8.33(\mathrm{br}, 2 \mathrm{H})$, 7.88-7.63 (br, 2H), 7.55-7.39 (br, 2H), 1.74-1.62 (br, 4H), 1.341.14 (br, 40H), 0.94-0.811 (br, 24H); ${ }^{13} \mathrm{C} \mathrm{NMR} \mathrm{(} \delta$ in $\mathrm{C}_{6} \mathrm{D}_{4} \mathrm{Cl}_{2}, 125$ $\mathrm{MHz}$ ) 37.56-37.06, 36.14-35.47, 30.16-28.90, 23.94-23.12, 15.02-14.23, 11.29-10.99, $\mathrm{sp}^{2}$ carbon signals could not be observed, likely due to the signal broadening.

Polymer pDTG2-PT was obtained as a black solid, in a fashion similar to that above. For Soxhlet extraction, hot benzene was used in place of hot toluene: m.p. $>300{ }^{\circ} \mathrm{C},{ }^{1} \mathrm{H}$ NMR ( $\delta$ in $\mathrm{CD}_{2} \mathrm{Cl}_{2}, 500 \mathrm{MHz}$ ) 8.80-8.50 (br m, 1H), 8.24-8.12 (br $\mathrm{m}, 1 \mathrm{H}), 7.28-7.06$ (br, 2H), 7.00-6,88 (br, 1H), 1.92-1.86 (br, 4H), 1.46-1.02 (br m, 40H), 1.00-0.74 (br, 24H), ${ }^{13} \mathrm{C}$ NMR $(\delta$ in $\left.\mathrm{C}_{6} \mathrm{D}_{4} \mathrm{Cl}_{2}, 125 \mathrm{MHz}\right) 37.51-37.10,36.03-35.66,29.38-28.87$, 23.51-23.20, 21.15-20.77, 14.52-14.26, 11.24-10.90, $\mathrm{sp}^{2}$ carbon signals could not be observed, likely due to the signal broadening.

\section{Results and discussion}

\section{Polymer synthesis}

A DTG2 monomer (DTG2Sn) was prepared by bromination of bis(trimethylsilyl)bi(dithienogermole) (DTG2Si) $^{8}$ with NBS yielding the dibromide (DTG2Br), followed by lithiation then stannylation, as presented in Scheme 1. Polymers with alternating DTG2 and BT or PT units were synthesized as black solids by Stille coupling of DTG2Sn and dibromobenzo- or pyridinothiadiazole, respectively, in refluxing chlorobenzene using $\mathrm{Pd}_{2}(\mathrm{dba})_{3} / \mathrm{P}(o-\mathrm{Tol})_{3} / \mathrm{CuO}$ as the catalyst (Scheme 1), similarly to what was previously reported for pDTG-BT $^{4 a}$ and pDTG-PT. ${ }^{6}$ Table 1 summarizes the polymer properties. pDTG2PT showed good solubility in chloroform, THF, and aromatic solvents, but insoluble in methanol, hexane, and ethyl acetate, e.g. $1.0 \mathrm{~g} \mathrm{~L}^{-1}$ in ODCB (o-dichlorobenzene). Whereas, pDTG2-BT was much less soluble. At room temperature, it was moderately soluble in ODCB (0.32 $\mathrm{g} \mathrm{L}^{-1}$ ) and CB (chlorobenzene), but insoluble in chloroform, THF, toluene, hexane, methanol, and ethyl acetate. The polymers exhibited good film forming properties by spin-coating to provide amorphous films. No XRD peaks were detected for the films, even after annealing and the AFM images showed smooth surface (Fig. S-2 and S-3†).

The molecular weight of pDTG2-PT was determined by GPC to be $M_{\mathrm{n}}=13000$ with polydispersity $\left(M_{\mathrm{w}} / M_{\mathrm{n}}\right)$ of 1.2 , relative to 

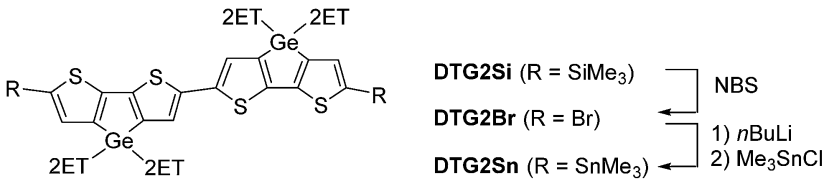

2ET = 2-ethylhexyl
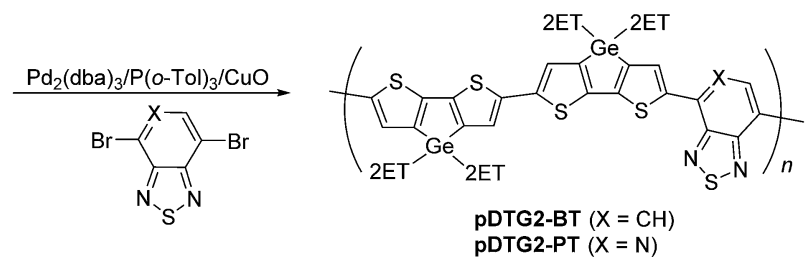

Scheme 1 Synthesis of DTG2-containing polymers.

polystyrene standards. How the GPC molecular weights of the present polymers correlate the real values is unclear. However, for a similar DTS polymer, the GPC data showed good agreement with that determined by the NMR spectroscopy. ${ }^{8}$ GPC analysis of pDTG2-BT could not be performed because of its low solubility. Our previous work indicated that pDTG-PT was less soluble than pDTG-BT, because of the higher polarity of PT than BT. In contrast to this, pDTG2-BT showed lower solubility than that of pDTG2-PT. This seems to be indicative of the higher molecular weight of pDTG2-BT than that of pDTG2-PT. The polymer structures were verified by ${ }^{1} \mathrm{H}$ and ${ }^{13} \mathrm{C}$ NMR spectra. Although the spectra revealed only broad signals, the proton integration ratios sufficiently agreed with the theoretical values (Fig. S-1†). Some unidentified signals with low intensities were also seen in the spectra. No stannyl end groups were detected in the spectra.

\section{Optical, electrochemical, and thermal properties}

UV-Vis absorption spectra of polymers pDTG2-BT and pDTG2PT were measured in CB solutions and as spin-coated films on quartz plates, revealing broad absorption bands, as depicted in Fig. 1. pDTG2-BT showed two absorption maxima. The major band at $738 \mathrm{~nm}$ reflects the interchain interactions in the aggregated polymer chains. The interactions enhance the polymer planarity and/or through space electronic $\pi-\pi$ interactions to lead to a low-energy shift of the band. On the other hand, the minor one at higher energy $\left(\lambda_{\max }=686 \mathrm{~nm}\right)$ is likely due to the aggregation-free polymer segments. Similar UV-Vis spectra have been previously reported for pDTS-BT $^{3 a}$ and pDTG-BT, ${ }^{4 a}$ and the appearance of red-shifted bands by the formation of aggregates by lowering the measurement temperature and evaporation of the solvent have been well studied for conjugated polymers. ${ }^{9}$ In fact, the lower-energy band of pDTG2BT was relatively intensified and the higher-energy band was weakened, when measured as a film. This is similar to the UVVis absorption properties of pDTG-BT, which reveals a shoulder peak at lower energy in addition to the major peak and the shoulder peak is intensified in film (Table 1). However, the aggregated band of pDTG-BT was observed only as a shoulder in the solution phase, indicating the stronger interchain interaction for pDTG2-BT. The aggregation-free band of pDTG2-BT was
Table 1 Properties of DTG2- and DTG-containing polymers

\begin{tabular}{llllll}
\hline & & \multicolumn{2}{l}{ UV-Vis Abs $\lambda_{\max }{ }^{a} / \mathrm{nm}$} & & \multirow{2}{*}{ TG } \\
\cline { 3 - 3 } Polymers & $M_{\mathrm{n}}\left(M_{\mathrm{w}} / M_{\mathrm{n}}\right)$ & In CB & As film & & $T_{\mathrm{d}}{ }^{5} /{ }^{\circ} \mathrm{C}^{b}$ \\
\hline pDTG2-BT & & 686,738 & 688,741 & 402 \\
pDTG2-PT & $13000(1.2)$ & 686 & 748 & 367 \\
pDTG-BT $^{c}$ & $15000(1.7)$ & $651,760^{d}$ & 687,760 & 401 \\
pDTG-PT $^{e}$ & $6200(1.5)$ & 745 & 739 & 405
\end{tabular}

${ }^{a}$ Data for minor bands and shoulders are given in Italic. ${ }^{b}$ In nitrogen. ${ }^{c}$ See ref. 4 a. ${ }^{d}$ In chloroform. ${ }^{e}$ See ref. 6.

red-shifted from that of pDTG-BT. This contrasts the results of computer simulation on polymer models in the gas phase, which shows similar electronic states for these polymers (vide infra).

As can be seen in Fig. 1, pDTG2-PT showed a broad single absorption peak at nearly the same energy as the minor peak of pDTG2-BT. This is also in marked contrast to that computation on their models provided a smaller band gap for the PTcontaining model (vide infra). Broadening of the absorption band of pDTG2-PT may be understood again by the existence of aggregated segments. However, it is likely that the interaction is not as strong as that in pDTG2-BT and the aggregated band is not clearly seen. Measuring the absorption spectrum of pDTG2PT as a film further broadened the band and moved the maximum to lower energy, indicating enhanced interchain interactions in film. Polymer pDTG-PT showed the absorption band at lower energy by approximately $60 \mathrm{~nm}$ in solution than that of pDTG2-PT with no evident spectral changes depending on the states (in solution or film). This is likely due to that the efficient interchain interactions took place for pDTG-PT even in the solution phase, arising from the highly polar pyridine units. However, for pDTG2-PT, the lower incorporation ratio of pyridine units in the polymer backbone leads to the relatively weak interaction in solutions and the considerable interactions are involved only in film. It was also demonstrated that pDTG-PT showed the major band at lower energy by approximately 100 $\mathrm{nm}$ than that of pDTG-BT, although pDTG-PT had a smaller molecular weight, presumably due to the more polar PT

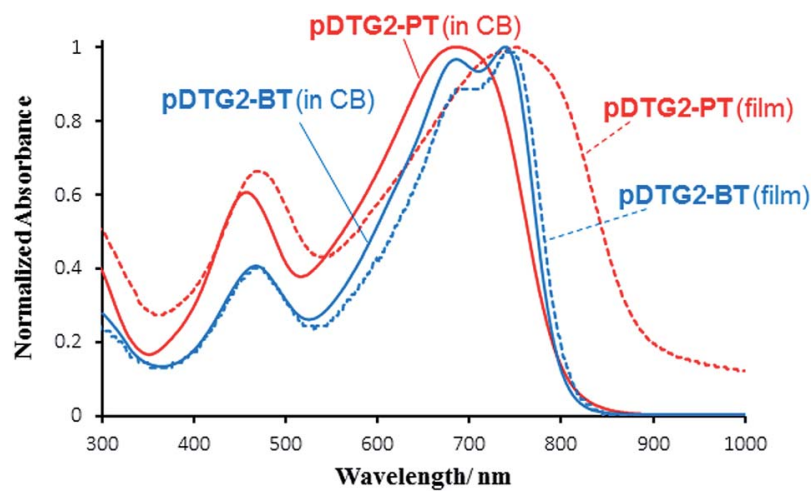

Fig. 1 UV-Vis absorption spectra of DTG2-containing polymers. 
structure which enhances the interchain interaction. However, in the present study, pDTG2-BT exhibited stronger interchain interaction than pDTG2-PT. Although we do not have any direct evidences, it is not unusual to assume that the stronger interaction of PDTG2-BT as compared to PDTG2-PT would be due to the higher molecular weight.

We also measured cyclic voltammograms (CVs) of the polymer films containing tetrabutylammonium perchlorate (TBAP) as a supporting electrolyte, as shown in Fig. 2. They showed pseudo-reversible anodic behaviors and pDTG2-PT showed the oxidation onset at higher potential, reflecting the stronger electron-withdrawing properties of PT as compared to BT, similarly to that the CV of pDTG-PT revealed a lower oxidation potential than that of pDTG-BT. ${ }^{5}$

Thermal stability of the polymers was investigated by TGA in nitrogen and the temperatures of $5 \%$ weight loss $\left(T_{\mathrm{d}}{ }^{5}\right)$ were noted as listed in Table 1. Thermal decomposition of the polymers occurred around $400{ }^{\circ} \mathrm{C}$, showing good thermal stability of the present polymers. The data are comparable to those of pDTG-BT ${ }^{4 a}$ and pDTG-PT, ${ }^{6}$ although $T_{\mathrm{d}}{ }^{5}$ value of pDTG2-PT was slightly lower than others.

\section{Quantum chemical calculations}

To understand the differences of electronic properties between pDTG-BT and pDTG2-BT, we carried out crystal orbital (CO) calculations on the polymer models simplified by the use of methyl groups as the substituents on the germanium atoms (pDTG-BT0 and pDTG2-BTo) at the level of B3LYP/6-31G(d) on the Gaussian 09 program. Optimized geometries of the unit cells, and HOCO and LUCO (highest occupied and lowest unoccupied $\mathrm{CO}$ ) profiles of the polymers are depicted in Fig. 3. For these polymers, several stable conformations may exist. With respect to the orientation of the sulphur atoms, anticonformation for the DTG-BT and DTG-DTG bonding seemed to be favourable as compared to the syn-conformation, because of the steric repulsion between the $\mathrm{C}-\mathrm{H}$ bonds of DTG and BT units for the syn-conformation. However, the syn-syn-anti-antiand syn-anti-anti-isomers presented in Fig. 3 were found to be slightly more stable than the all-anti isomers for pDTG-BT0 and pDTG2-BT0, respectively. The polymer optimized geometries show high planarity with the interplane angles less than $1^{\circ}$.

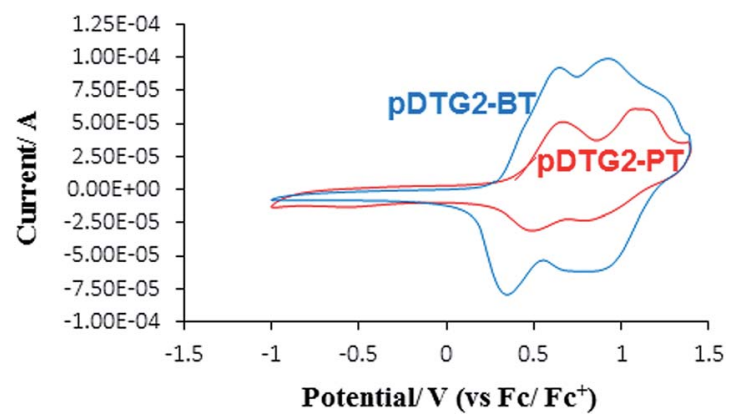

Fig. 2 CVs of films of DTG2-polymers containing TBAP as supporting electrolyte (polymer:TBAP $=1: 10$ in weight).
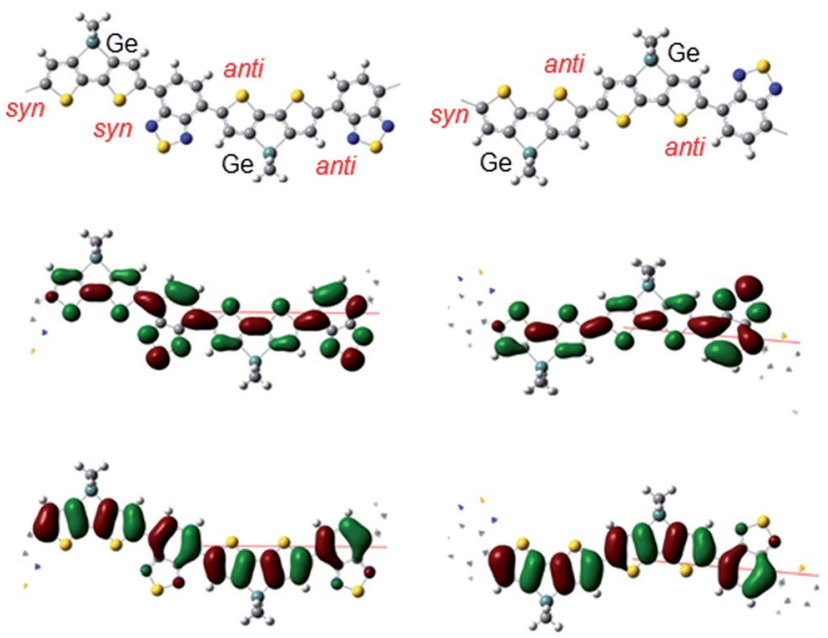

Fig. 3 Optimized geometries (top), LUCO (middle) and HOCO (bottom) profiles of unit cells of model polymers pDTG-BTO (left) and pDTG2-BT0 (right) with methyl groups on Ge atoms, derived from DFT calculations at B3LYP/6-31G(d).

The HOCO and LUCO energy levels and the band gaps derived from the calculations are summarized in Table 2 . Reasonably, both the HOCO and LUCO are destabilized as increasing the incorporation ratio of the electron rich DTG units in the polymer chain from DTG/BT $=1 / 1$ in PDTG-BT0 to $2 / 1$ in pDTG2-BT0. The band gap energy of pDTG-BT0 was calculated to be slightly smaller than that of PDTG2-BT0, disagreeing with the UV-Vis absorption data, i.e. the aggregation-free band of pDTG-BT appeared at higher energy by $35 \mathrm{~nm}$ than that of pDTG2-BT (Table 1). This may be due to the existence of large 2ethylhexyl substituents on the germanium atoms in PDTG2-BT. The substituents may cover the DTG2 plane to restrict the rotation around the DTG-DTG bonds, enhancing the planarity. Whereas, the DTG-BT bonds seem to be less affected by the substituents, thus being able to more freely rotate. Consequently, pDTG2-BT would possess higher planarity in the polymer chain. A model of pDTG2-PT (pDTG2-PT0) was also calculated, again revealing high planarity of the $\pi$-conjugated system (Fig. S-4 $\dagger$ ). The lower HOCO and LUCO energy levels and the smaller band gap than those of pDTG2-BT0 as listed in Table 2 arise from the stronger D-A interaction between DTG2 and PT than that between DTG2 and BT. However, in the real system, the aggregation-free bands of PDTG2-BT and pDTG2-PT appeared at nearly the same wavelength, presumably due to the larger molecular weight of pDTG2-BT, which balances the stronger D-A interaction in PDTG2-PT.

Table 2 HOCO and LUCO energy levels and band gaps for model polymers, derived from DFT calculations at B3LYP/6-31G(d)

\begin{tabular}{llll}
\hline Polymers & $\mathrm{HOCO} / \mathrm{eV}$ & $\mathrm{LUCO} / \mathrm{eV}$ & Band gap/eV \\
\hline pDTG-BT0 & -4.43 & -2.96 & 1.47 \\
pDTG2-BT0 & -4.33 & -2.81 & 1.52 \\
pDTG2-PT0 & -4.42 & -3.03 & 1.38
\end{tabular}




\section{Photovoltaic and semiconducting properties}

BHJ-PSCs using the present polymers as the host materials and $\mathrm{PC}_{71} \mathrm{BM}$ as the guest were fabricated with the structure of ITO (150 nm)/PEDOT:PSS (30 nm)/pDTG2-polymer:PC ${ }_{71} \mathrm{BM}(1: 2.5$ wt ratio, ca. $80 \mathrm{~nm}) / \mathrm{LiF}(0.5 \mathrm{~nm}) / \mathrm{Al}(80 \mathrm{~nm})$ and an active area of $0.25 \mathrm{~cm}^{2}$, in a fashion similar to what was reported previously. ${ }^{6}$ As expected, the cells showed clear photovoltaic properties (Fig. 4). Annealing the active layers as well as the devices did not affect the results. The photovoltaic parameters of the cells are listed in Table 3 in comparison with those of pDTG-BT $^{\mathbf{a} a}$ and pDTG-PT, ${ }^{6}$ reported previously. Notably, the cell with pDTG2-BT exhibited higher performance than the one with pDTG2-PT. Although the open-circuit voltage $\left(V_{\text {oc }}\right)$ of the pDTG2-BT-based cell is lower than that based on pDTG2-PT, due to the higher lying HOMO (Highest Occupied Molecular Orbital) of pDTG2BT, its larger current density efficiently raises the power conversion efficiency (PCE). It is most likely that the higher degree of interchain interaction of pDTG2-BT is responsible for the higher current density. The PCE of pDTG2-BT-based device is also higher than the similar devices with pDTG-BT (PCE = $1.21 \%)^{4 a}$ and pDTG-PT $(\mathrm{PCE}=2.92 \%),{ }^{6}$ reported previously by us.

Spin-coated films of the present polymers were investigated as p-type semiconducting materials (Fig. S-5 $\dagger$ ) and the data are also listed in Table 3. Polymer pDTG2-BT shows higher mobility than that of pDTG2-PT, in accordance with the higher $J_{\mathrm{sc}}$ for
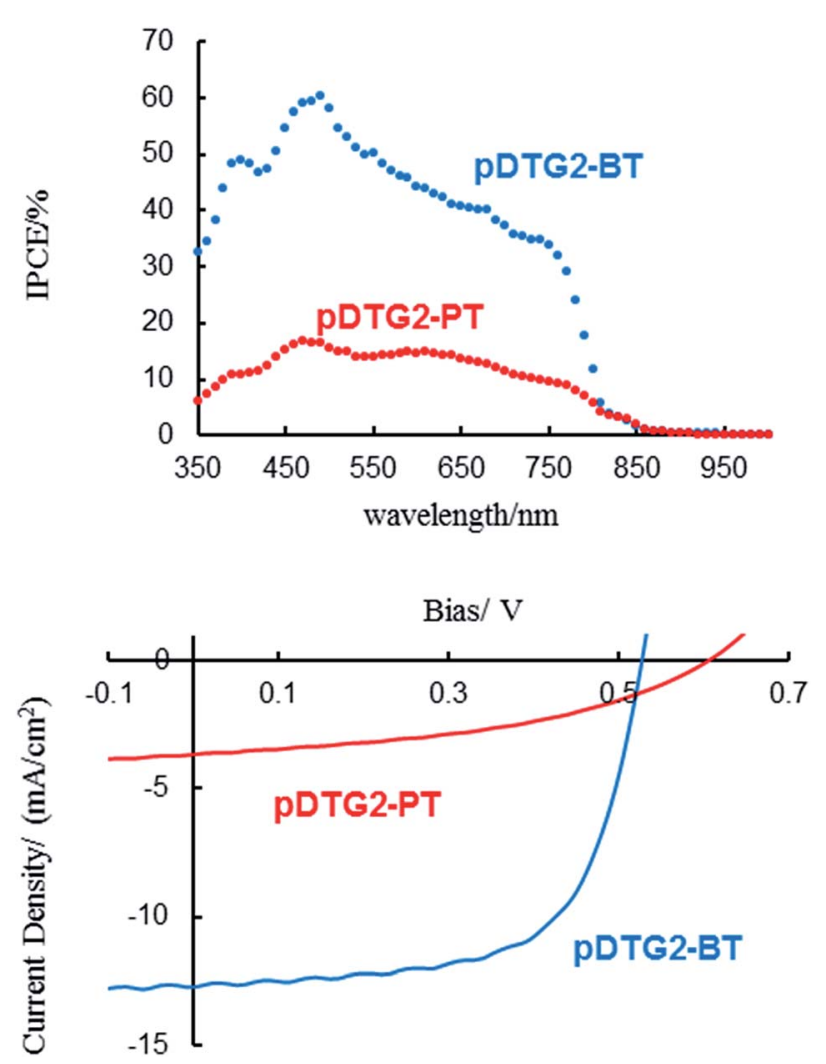

Fig. 4 IPCE (incident photon-to-current conversion efficiency) (top) and J-V (bottom) characteristics of BHJ-PSCs based on DTG2-containing polymers.
Table 3 PSC and TFT parameters based on DTG2-polymers

\begin{tabular}{|c|c|c|c|c|c|c|}
\hline \multirow[b]{2}{*}{ Polymers } & \multicolumn{4}{|l|}{ PSC } & \multicolumn{2}{|l|}{ TFT } \\
\hline & $\begin{array}{l}J_{\mathrm{sc}} / \\
\mathrm{mA} \mathrm{cm}\end{array}$ & $\begin{array}{l}V_{\mathrm{oc}} l \\
\mathrm{~V}\end{array}$ & $\mathrm{FF}$ & $\begin{array}{l}\text { PCE/ } \\
\%\end{array}$ & $\begin{array}{l}\mu_{\mathrm{FET}} / \\
\mathrm{cm}^{2} \mathrm{~V}^{-1} \mathrm{~s}^{-1}\end{array}$ & $\begin{array}{l}I_{\text {on }} / \\
I_{\text {off }}\end{array}$ \\
\hline pDTG2-BT & 12.69 & 0.53 & 0.64 & 4.30 & $3.8 \times 10^{-3}$ & $10^{5}$ \\
\hline pDTG2-PT & 3.66 & 0.60 & 0.43 & 0.94 & $1.4 \times 10^{-4}$ & $10^{4}$ \\
\hline
\end{tabular}

pDTG2-BT-based PSC. It is also noted that the mobility of pDTG2-BT is approximately 10 times higher than that of pDTS2BT $\left(\mu_{\mathrm{FET}}=3 \times 10^{-4} \mathrm{~cm}^{2} \mathrm{~V}^{-1} \mathrm{~s}^{-1}\right)$, reported previously. ${ }^{7}$ This may be due to the film morphology. However, no evident differences were observed in the AFM images of the films (Fig. S$3 \dagger)$, thus being attributable primarily to the enhanced polymer interchain interaction in the film for pDTG2-BT.

\section{Conclusions}

We successfully prepared two D-A polymers containing DTG2 as the donor and applied them as BHJ-PCS and TFT materials. Although quantum chemical calculations on their models showed that DTG2-polymers should have similar electronic structures to those of the corresponding DTG-polymers with slightly larger band gaps, the pDTG2-BT-based PSC exhibited remarkably higher performance than those of pDTG-BT-based one, reported previously. ${ }^{4 a}$ It is also noteworthy that pDTG2-BT showed superior performance as the active material for TFT to pDTS2-BT, indicating the potential of DTG2. This is likely due to high planarity of the DTG2 unit. In addition, high solubility of the DTG2-containing polymers arising from four solubilizing groups on the germanium atoms seems to improve the filmforming properties as compared to DTG-containing ones, thus being responsible for the enhanced performance. The results demonstrated in this paper clearly shows the potential of DTG2 as the donor component of D-A polymers, providing opportunities to design new functional materials.

\section{Acknowledgements}

This work was supported by a Grant-in-Aid for Scientific Research (B) (no. 26288094) from the Ministry of Education, Culture, Sports, Science and Technology, Japan. We also thank Prof. Kazuyoshi Tanaka and Prof. Hiroyuki Fueno for the assistance in crystal orbital calculations.

\section{Notes and references}

1 For a recent review, see: Y.-F. Lee, Acc. Chem. Res., 2012, 45, 723.

2 (a) J. Ohshita, Macromol. Chem. Phys., 2001, 210, 1360; (b) J. Ohshita, M. Nodono, T. Watanabe, Y. Ueno, A. Kunai, Y. Harima, K. Yamashita and M. Ishikawa, J. Organomet. Chem., 1998, 553, 487; (c) J. Ohshita, M. Nodono, H. Kai, T. Watanabe, A. Kunai, K. Komaguchi, M. Shiotani, 
A. Adachi, K. Okita, Y. Harima, K. Yamashita and M. Ishikawa, Organometallics, 1999, 18, 1453.

3 (a) J. Hou, H.-Y. Chen, S. Zhang, G. Li and Y. Yang, J. Am. Chem. Soc., 2008, 130, 16144; (b) L. Huo, H.-Y. Chen, J. Hou, T. L. Chen and Y. Yang, Chem. Commun., 2009, 5570; (c) M. Zhang, X. Guo, Z.-G. Zhang and Y. Li, Polymer, 2001, 52, 5464; (d) J. Peet, L. Wen, P. Byrne, S. Rodman, K. Forberich, Y. Shao, N. Drolet and R. Gaudiana, Appl. Phys. Lett., 2011, 98, 043301; (e) S. Subramaniyan, H. Xin, F. S. Kim, S. Shoaee, J. R. Durrant and S. A. Jenekhe, Adv. Energy. Mater., 2011, 1, 854; (f) S. Subramaniyan, H. Xin, F. S. Kim and S. A. Jenekhe, Macromolecules, 2011, 44, 6245; $(g)$ T.-Y. Chu, J. Lu, S. Beaupre, Y. Zhang, J.-R. Pouliot, S. Wakim, J. Zhou, M. Leclerc, Z. Li, J. Ding and Y. Tao, J. Am. Chem. Soc., 2011, 133, 4250; (h) Y. Ie, J. Huang, Y. Uetani, M. Karakawa and Y. Aso, Macromolecules, 2012, 45, 4564; (i) X. Guo, N. Zhou, S. Lou, J. W. Hennek, R. P. Ortiz, M. R. Butler, P.-L. Boudreault, J. Strzalka, P.-O. Morin, M. Leclerc, J. T. L. Navarrete, M. A. Ratner, L. X. Chen, P. H. Chang, A. Facchetti and T. J. Marks, J. Am. Chem. Soc., 2012, 134, 18427.

4 (a) J. Ohshita, Y.-M. Hwang, T. Mizumo, H. Yoshida, Y. Ooyama, Y. Harima and Y. Kunugi, Organometallics, 2011, 30, 3233; (b) Y.-M. Hwang, J. Ohshita, Y. Harima, T. Mizumo, Y. Ooyama, Y. Morihara, T. Izawa, T. Sugioka and A. Fujita, Polymer, 2011, 52, 3912; (c) C. M. Amb, S. Chen, K. R. Graham, J. Subbiah, C. E. Small, F. So and J. R. Reynolds, J. Am. Chem. Soc., 2011, 133, 10062; (d) D. Gendron, P.-O. Morin, P. Berrouard, N. Allard, B. R. Aïch, C. N. Garon, Y. Tao and M. Leclerc, Macromolecules, 2011, 44, 7188.

5 (a) C. E. Small, S. Chen, J. Subbiah, C. M. Amb, S. W. Tsang, T. H. Lai, J. R. Reynolds and F. So, Nat. Photonics, 2012, 6, 115; (b) C. P. Yau, Z. Fei, R. S. Ashraf, M. Shahid, S. E. Watkins, P. Pattanasattayavong, T. D. Anthopoulos, V. G. Gregoriou, C. L. Chohos and M. Heeney, Adv. Mater., 2014, 24, 678.

6 J. Ohshita, M. Miyazaki, F.-B. Zhang, D. Tanaka and Y. Morihara, Polym. J., 2013, 45, 979.

7 P. M. Beaujuge, W. Pisula, H. K. Tsao, S. Ellinger, K. Müllen and J. R. Reynolds, J. Am. Chem. Soc., 2009, 131, 7514.

8 J. Ohshita, M. Miyazaki, D. Tanaka, Y. Morihara, Y. Fujita and Y. Kunugi, Polym. Chem., 2013, 4, 3116.

9 For example, see: (a) S. Yue, G. C. Berry and R. D. McCullough, Macromolecules, 1996, 29, 933; (b) C.-Z. Zhou, T. Liu, T.-T. Lin, X.-H. Zhan and Z.-K. Chen, Polymer, 2005, 46, 10952; (c) J. Clark, C. Silva, R. H. Friend and F. C. Spano, Phys. Rev. Lett., 2007, 98, 206406; (d) S. T. Turner, P. Pingel, R. Steyrleuthner, E. J. W. Crossland, S. Ludwig and D. Neher, Adv. Funct. Mater., 2011, 21, 4640. 